\title{
Proteasomes in immune cells: more than peptide producers?
}

\author{
Marcus Groettrup, Christopher J. Kirk and Michael Basler
}

Abstract |When cells are stimulated with pro-inflammatory cytokines, most of their constitutively expressed proteasomes are replaced with immunoproteasomes, which increase the production of peptides for presentation on MHC class I molecules. In addition, cortical thymic epithelial cells selectively express a type of proteasome known as the thymoproteasome that is required for the positive selection of thymocytes. Here, we discuss how these specialized types of proteasome shape the $T$ cell receptor repertoire of cytotoxic $T$ lymphocytes and propose that immunoproteasomes have functions, in addition to antigen processing, that influence cytokine production and $T$ cell differentiation, survival and function. We also discuss how inhibitors of immunoproteasomes can suppress undesired $\mathrm{T}$ cell responses in autoimmune diseases.

Antigen recognition by cytotoxic T lymphocytes (CTLs) occurs through the interaction of their T cell receptors (TCRs) with peptideMHC class I complexes. The peptides presented by MHC class I molecules are derived either from endogenous proteins in the direct presentation pathway or from proteins taken up from the extracellular environment during cross-presentation ${ }^{1}$. In both direct and cross-presentation pathways, the proteasome is the protease that determines the carboxy-terminal anchor residues of MHC class I-binding peptides. The proteasome produces peptides of $8-9$ amino acids that can bind directly to the peptide-binding cleft of MHC class I molecules and it also produces amino-terminally extended precursor peptides that are processed further by aminopeptidases in the cytoplasm or endoplasmic reticulum ${ }^{2}$ (FIG. 1).

The proteasome is an evolutionarily ancient enzyme and is present in a simplified form in archaebacteria ${ }^{3}$. Because of the evolutionary conservation of the proteasome, it has been proposed that the entire MHC class Irestricted antigen presentation pathway has evolved to accommodate the peptides that the proteasome generates ${ }^{4}$. The proteasome consists of a central proteolytic unit, known as the
$20 \mathrm{~S}$ proteasome, and the $19 \mathrm{~S}$ regulator, which together make up a $26 \mathrm{~S}$ structure ${ }^{5}$. Moreover, the interferon- $\gamma(\mathrm{IFN} \gamma)$-inducible heteroheptameric regulator proteasome activator 28 (PA28) which is composed of PA28a (also known as PSME1) and PA28ß (also known as PSME2) subunits ${ }^{6,7}$, can associate with the $26 \mathrm{~S}$ proteasome to form the 'hybrid' proteasome ${ }^{8}$. Evidence from mutant cell lines ${ }^{9,10}$ and mice ${ }^{11}$ shows that PA28 influences antigen processing by either affecting peptide cleavage ${ }^{12,13}$ or facilitating the release of peptide products from the proteasome complex ${ }^{14}$.

The cylindrical 20S proteasome consists of four heteroheptameric rings: two outer rings composed of seven $\alpha$-type structural subunits and two inner rings composed of seven $\beta$-type structural and proteolytic subunits. Most mammalian tissues express 'constitutive' proteasomes, in which the proteolytic activity is mediated by proteasome subunit $\beta 1$ (also known as PSMB6, Y and $\delta$ ), which cleaves after acidic residues (caspase-like activity), proteasome subunit $\beta 2$ (also known as PSMB7, $\mathrm{Z}$ and MC14), which cleaves after basic residues (trypsin-like activity), and proteasome subunit $\beta 5$ (also known as PSMB5, $X$, $\mathrm{MB} 1$ and $\varepsilon$ ), which cleaves after hydrophobic residues (chymotrypsin-like activity) (FIG. 2).
With the exception of $\beta 5$, the proteolytic activities of the constitutive proteasome subunits do not fully match the requirements for the generation of MHC class I ligands. Human MHC class I molecules accommodate peptides with hydrophobic residues (products of $\beta 5$-mediated cleavage) and occasionally basic residues (products of $\beta 2$-mediated cleavage) at their C-termini, whereas mouse MHC class I molecules only accommodate peptides with hydrophobic C-terminal residues. Peptides with acidic C-terminal residues (products of $\beta 1$-mediated cleavage) have an inappropriate C-terminus and cannot function as MHC class I ligands in mice or humans ${ }^{15}$.

In the early 1990s, two additional $\beta$-type proteasome subunits, designated proteasome subunit $\beta 1 \mathrm{i}$ (also known as PSMB9 and LMP2) and proteasome subunit $\beta 5 \mathrm{i}$ (also known as PSMB8 and LMP7) were identified $^{16-19}$. These subunits, which are highly homologous to $\beta 1$ and $\beta 5$, respectively, are encoded by genes in the MHC class II region adjacent to the genes encoding transporter associated with antigen processing 1 (TAP1) and TAP2, and the expression of $\beta 1 \mathrm{i}$ and of $\beta 5 \mathrm{i}$ are strongly and synergistically induced by the proinflammatory cytokines IFN $\gamma$ and tumour necrosis factor $(\mathrm{TNF})^{20}$. Subsequently, another cytokine-inducible proteasome subunit with homology to $\beta 2$, proteasome subunit $\beta 2$ i (also known as PSMB10, LMP10 and MECL1), was found outside the MHC region $^{21-23}$. After stimulation with IFN $\gamma$ and/or TNF, expression of these three inducible 'immunosubunits' is strongly upregulated and the neosynthesis of $20 \mathrm{~S}$ proteasomes is switched almost exclusively to the generation of a type of proteasome known as the immunoproteasome $e^{24,25}$. Indeed, by eight days after infection of mice with a virus, bacterium or fungus, constitutive proteasomes in the liver and other tissues are almost completely replaced by immunoproteasomes ${ }^{26,27}$. However, despite nearly two decades of research, the specific reasons for this exchange of proteasome subunits are not completely understood.

The pool of MHC class I ligands that is generated by the immunoproteasome is both distinct from and more efficient at 


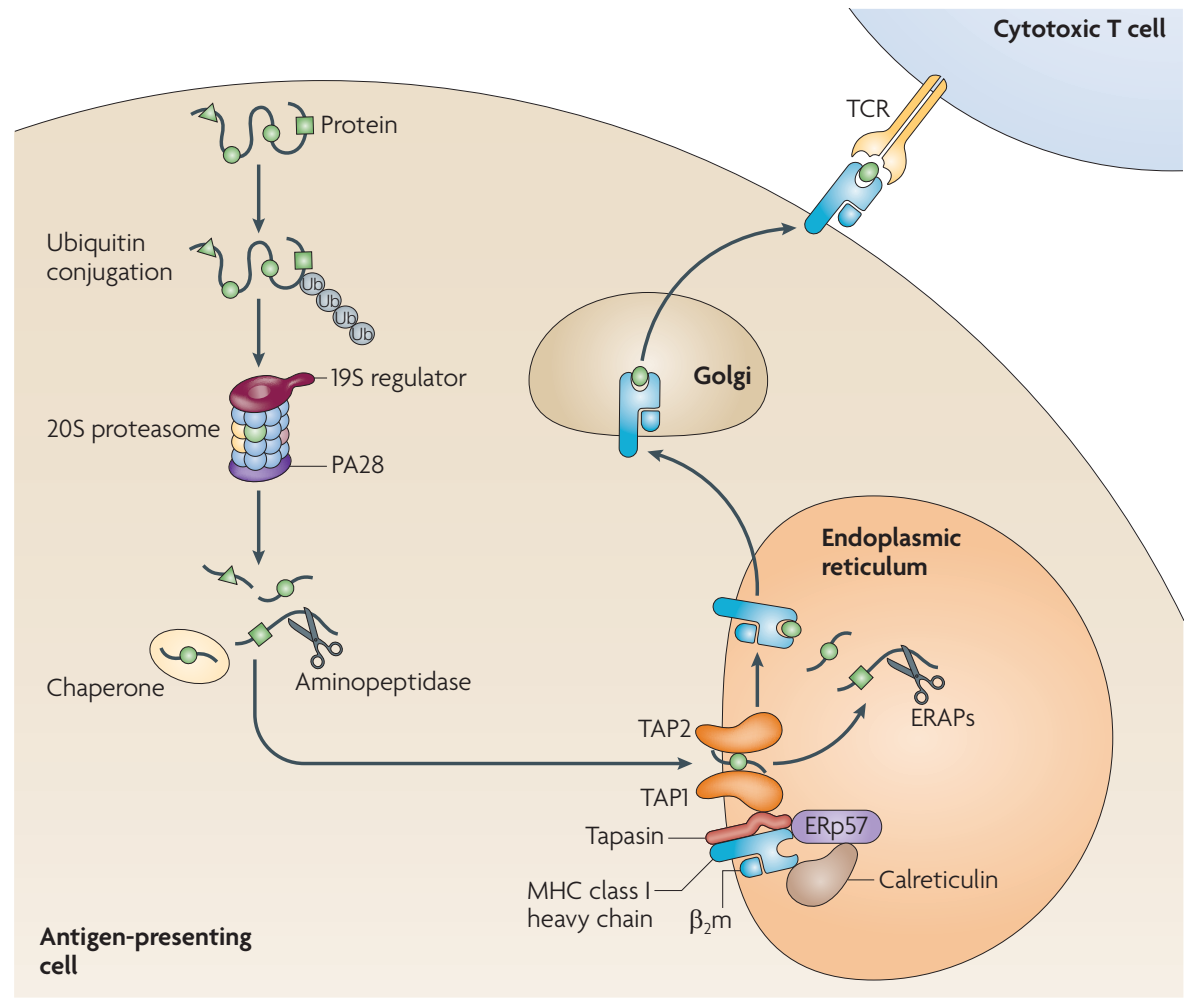

Figure 1 | Antigen processing in the MHC class I-restricted pathway. Proteins that are synthesized in the cell (direct presentation) or are released from endosomes (cross-presentation) are polyubiquitylated in the cytoplasm and degraded by hybrid proteasomes consisting of the $20 \mathrm{~S}$ proteasome core, the $19 \mathrm{~S}$ regulator and PA28. The peptides that are produced are either of the ideal length for binding to MHC class I molecules (8-9 amino acids) or are amino-terminally extended precursors that can be further cleaved by aminopeptidases in the cytoplasm (such as leucine aminopeptidase, puromycin-sensitive aminopeptidase, bleomycin hydrolase and tripeptidyl peptidase II). Chaperones (such as heat shock protein 70 (HSP70), HSP90 $\alpha$ and TRiC) can stabilize the peptides in the cytoplasm to prevent their rapid degradation (for example by tripeptidyl peptidase II or thimet oligopeptidase). Transporter associated with antigen processing 1 (TAP1) and TAP2, which are attached to nascent MHC class I chains through tapasin, transport the peptides into the endoplasmic reticulum (ER), where they can be further trimmed at the $\mathrm{N}$-terminus by ER aminopeptidase 1 (ERAP1) and ERAP2. The oxidoreductase ERp57 ensures the maintenance of disulphide bridges in the MHC class I loading complex. Note that the carboxyl terminus of a peptide ligand for $\mathrm{MHC}$ class I molecules is mainly determined by proteasomal cleavage. The binding of peptides with high affinity to the MHC class I heavy chain- $\beta_{2}$-microglobulin $\left(\beta_{2} \mathrm{~m}\right)$ complex induces a final folding and release of the MHC class I molecule from the ER lumenal chaperone calreticulin to allow exit from the ER and migration through the Golgi to the plasma membrane. TCR, T cell receptor.

CTL activation than the ligand pool generated by the constitutive proteasome ${ }^{28,29}$. This is a result, in part, of the replacement of $\beta 1$ with $\beta 1$, which leads to the elimination of the caspase-like activity of $\beta 1$ and enhancement of the chymotrypsin-like activity of $\beta 1 \mathrm{i}$ and therefore to the generation of peptides with hydrophobic C-terminal residues ${ }^{25,30-34}$ Mice lacking one or more of the inducible immunosubunits have been generated and infected with commonly used laboratory strains of viruses, bacteria and fungi ${ }^{35,36}$. These studies have indicated roles for the immunoproteasome in shaping the CTL repertoire and in pathogen clearance that have, until recently (see later), been ascribed to alterations in the MHC class I ligands that are generated.
In cortical thymic epithelial cells (cTECs), which are involved in the positive selection of $\mathrm{T}$ cells in the thymus, a third type of specialized proteasome (the thymoproteasome) has been discovered that, in addition to the immunosubunits $\beta 1 \mathrm{i}$ and $\beta 2 \mathrm{i}$, also contains the cTEC-specific proteasome subunit $\beta 5$ t (also known as PSMB11), which seems to be essential for the positive selection of $\mathrm{CD}^{+} \mathrm{T}^{\mathrm{T}}$ cells ${ }^{37}$.

Immunoproteasomes and thymoproteasomes are thought to function in shaping CTL responses at the level of antigen presentation. In this article, we review these insights into the biology of immune-associated proteasomes and propose, based on recent data, that immunoproteasomes also have a role in the control of cytokine production and $\mathrm{T}$ cell differentiation. The therapeutic implications of these immunoproteasome functions that are independent of antigen processing in immune responses are also discussed.

\section{Immunoproteasome-deficient mice}

The discovery of the genes encoding $\beta 1 \mathrm{i}$ and $\beta 5 \mathrm{i}$ in the MHC region led researchers to assume that they would have important functions in the immune response, but initial functional and phenotypic analyses of knockout mice were disappointing. $\beta 1$ i-deficient mice generated normal CTL responses to Sendai virus and to ovalbumin and cleared lymphocytic choriomeningitis virus (LCMV) infection; CTL responses to the LCMV epitopes GP33, GP276 and NP396 were unaltered in these mice ${ }^{38}$. The CTL response to influenza virus infection in $\beta 1$ i-deficient mice was skewed towards the sub-dominant epitopes of the virus (PB1F2.61 and NS2.114) and away from two immunodominant epitopes (NP366 and PA224) ${ }^{39}$. Despite the initial report that splenocytes from $\beta 5 \mathrm{i}$ deficient mice generate a decreased number of CTLs specific for the male minor antigen $\mathrm{HY}^{36}$, we observed normal responses to all dominant LCMV epitopes and normal kinetics of viral clearance in $\beta 5 i$-deficient mice ${ }^{38}$. However, after challenge with recombinant vaccinia virus or a DNA vaccine encoding the LCMV glycoprotein, an increased response to the LCMV epitope GP276 was detected in $\beta 5$ i-deficient mice, which indicates that the immunoproteasome downregulates the presentation of this epitope in wild-type mice ${ }^{38,40}$.

A role for $\beta 5 \mathrm{i}$ in the clearance of pathogens was first shown in knockout mice after infection with Listeria monocytogenes ${ }^{41}$. Similar to LCMV infection, infection with L. monocytogenes results in the upregulation of immunoproteasome expression and the replacement of constitutive proteasomes in the liver ${ }^{26}$. Although L. monocytogenesspecific CTLs were generated at a normal frequency in $\beta 5$ i-deficient mice, the clearance of bacteria from the liver was not apparent by day 10 , by which time bacterial burden in the spleen of wild-type mice had decreased ${ }^{41}$. This result underscores the necessity to induce immunoproteasomes at sites of infection for pathogen clearance, most probably because the effective CTL response is focused on immunoproteasome-dependent pathogen epitopes.

An even more prominent phenotype was seen when $\beta 5$ i-deficient mice were infected with the protozoan parasite Toxoplasma gondii ${ }^{42}$. In contrast to wild-type mice, $\beta 5 \mathrm{i}$ deficient mice succumbed to infection and 


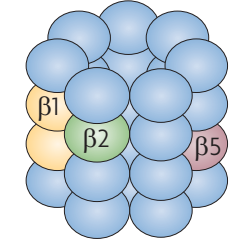

Constitutive proteasome

$\beta 1$ (PSMB6, Y, $\delta$ )

$\beta 2$ (PSMB7, Z, MC14)

$\beta 5(\mathrm{PSMB} 5, \mathrm{X}, \mathrm{MB} 1, \varepsilon)$

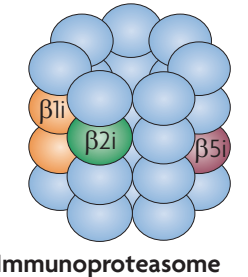

B1i (PSMB9, LMP2)

$\beta 2 \mathrm{i}$ (PSMB10, LMP10, MECL1)

$\beta 5 \mathrm{i}$ (PSMB8, LMP7)

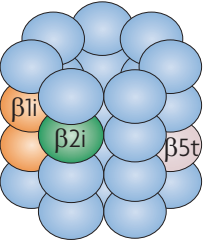

Thymoproteasome

Bli (PSMB9, LMP2)

$\beta 2 \mathrm{i}$ (PSMB10, LMP10, MECL1)

$\beta 5 t$ (PSMB11)

Figure 2 | Subunit composition of the active sites of the constitutive proteasome, immunoproteasome and thymoproteasome. The proteolytic subunits of the constitutive proteasome are $\beta 1$ (also known as PSMB6, $Y$ and $\delta$ ), $\beta 2$ (also known as PSMB7, Z and MC14) and $\beta 5$ (also known as PSMB5, X, MB1 and $\varepsilon$ ). The proteolytic immunoproteasome subunits are $\beta 1 \mathrm{i}$ (also known as PSMB9 and LMP2), $\beta 2 i$ (also known as PSMB10, LMP10 and MECL1) and $\beta 5 i$ (also known as PSMB8 and LMP7). The proteolytic thymoproteasome subunits are $\beta 1 \mathrm{i}, \beta 2 \mathrm{i}$ and $\beta 5 \mathrm{t}$ (also known as PSMB11). Compared with the constitutive proteasome, the immunoproteasome has a strongly decreased caspase-like activity and an increased chymotrypsin-like activity, whereas the thymoproteasome has a decreased chymotrypsin-like activity.

this correlated with decreased production of IFN $\gamma$ by parasite-specific $\mathrm{CD} 8^{+} \mathrm{T}$ cells. Immunodominant epitopes of the T. gondiispecific CTL response have not been identified and it remains to be shown whether the decreased generation of activated CTLs in $\beta 5$ i-deficient mice is a result of the lack of presentation of $\beta 5 \mathrm{i}$-dependent $T$. gondii epitopes or whether additional functions of $\beta 5 \mathrm{i}$ might explain this phenotype. Taken together, these results show that the requirement for immunoproteasomes for pathogen elimination varies markedly between infection models and further testing of immunoproteasome-deficient mice is required to fully appreciate the contribution of individual proteasome subunits to the immune response to infectious agents.

Stimulation of cells with IFN $\gamma$ or TNF typically leads to a tenfold upregulation of the cell surface expression of MHC class I molecules ${ }^{43}$. Through increased ligand production, switching to the generation of immunoproteasomes provides the larger pool of peptides that is required to allow the increased number of MHC class I molecules to finalize their folding in the endoplasmic reticulum and to migrate to the cell surface. Evidence of a role for $\beta 5 \mathrm{i}$ in contributing to the increased cell surface expression of MHC class I molecules has been obtained in $\beta 5 \mathrm{i}-$ deficient mice, which have a $50 \%$ decrease in cell surface expression of MHC class I molecules by lymphocytes and monocytes compared with wild-type mice ${ }^{36}$. Remarkably, no decrease in the cell surface expression of MHC class I molecules has been observed in $\beta 1$ i- or $\beta 2 \mathrm{i}$-deficient mice $\mathrm{e}^{35,44}$. The normal level of MHC class I expression on the cell surface of $\beta 1 \mathrm{i}$ - but not $\beta 5 \mathrm{i}$-deficient splenocytes (as determined by flow cytometry) is surprising as $\beta 1 \mathrm{i}$ contributes to the generation of MHC class I-binding peptides by replacing the caspase-like activity of $\beta 1$ with the chymotrypsin-like activity of $\beta 1$. These data indicate that $\beta 5 \mathrm{i}$ produces higher affinity MHC class I ligands than does $\beta 5$, which was not predicted from structural models of the two subunits ${ }^{33}$.

Decreased cell surface MHC class I expression might be predicted to affect the number of $\mathrm{CD}^{+} \mathrm{T}$ cells in $\beta 5 \mathrm{i}$-deficient mice. However, a surprising finding was a $20-30 \%$ decrease in the number of $\mathrm{CD} 8^{+} \mathrm{T}$ cells compared with $\mathrm{CD}^{+} \mathrm{T}$ cells in the thymus, blood and spleen of $\beta 1 \mathrm{i}$ - or $\beta 2 \mathrm{i}$-deficient mice, but not $\beta 5 \mathrm{i}$-deficient mice ${ }^{35,45}$. As these findings did not correlate with MHC class I expression levels, it is possible that they were a result of intrinsic T cell effects. Indeed, this has been elegantly shown using bone marrow chimaeras in which wild-type recipient mice received equal numbers of cells from wild-type and from $\beta 5 i$ and $\beta 2 i$ double-deficient donors. The decreased ratio of $\mathrm{CD}^{+}$to $\mathrm{CD} 4^{+} \mathrm{T}$ cells was maintained in the thymus and periphery for $\beta 5 \mathrm{i}$ and $\beta 2 \mathrm{i}$ double-deficient donor cells in wild-type recipient mice, whereas wild-type donor cells had normal numbers of both cell subsets $^{46}$. As the two donor populations were selected by the same wild-type thymus and expanded in the same wild-type periphery, this phenomenon cannot be attributed to a difference in antigen presentation. Rather, it seems that $\mathrm{CD}^{+} \mathrm{T}$ cells deficient in $\beta 5 \mathrm{i}$ and $\beta 2 \mathrm{i}$ expand less readily than wild-type $\mathrm{CD} 8^{+}$ $\mathrm{T}$ cells. This finding clearly hints at a previously overlooked function of immunoproteasome subunits in the proliferative expansion of $\mathrm{CD}^{+} \mathrm{T}$ cells.

\section{TCR repertoire formation}

The cells that are responsible for negative selection and TCR repertoire formation in the thymus - thymic dendritic cells and medullary thymic epithelial cells (mTECs) constitutively express high levels of immunoproteasomes (FIG. 3). By contrast, cTECs, which support the positive selection of T cells, also express immunoproteasome subunits but only after systemic infection or the administration of IFN $\gamma^{47}$. Therefore, it is not surprising that immunoproteasomes shape the repertoire of $\mathrm{CD}^{+} \mathrm{T}$ cells in the thymus. It has been shown that the lack of NP366specific CTLs in $\beta 1$ i-deficient mice infected with influenza virus is not owing to the inability of $\beta 1$ i-deficient splenocytes to present the epitope for the activation of CTLs, as was previously reported ${ }^{35}$, but instead is owing to the lack of NP366-specific precursor T cells in the periphery of knockout mice ${ }^{39}$. In addition, $\beta 2$ i-deficient mice infected with LCMV mounted a normal CTL response to most LCMV epitopes but the response to GP276 was markedly decreased ${ }^{44}$. Again, this defect was not caused by the inability of $\beta 2$ i-deficient antigen-presenting cells (APCs) to process and present the GP276 epitope to CTLs but by a decrease in the number of GP276-specific precursor T cells in the knockout mice.

Differences in T cell selection in the thymus can be best observed in TCR-transgenic mice. $\mathrm{CD}^{+} \mathrm{T}$ cells from OT-1 mice, which express a TCR that is specific for the ovalbumin epitope SIINFEKL presented by the MHC class I molecule $\mathrm{H}-2 \mathrm{~K}^{\mathrm{b}}$, did not undergo positive selection in the absence of $\beta 5 \mathrm{i}$ (REF. 48). In vitro processing of the $\mathrm{Cpal}_{92-99}$ self peptide derived from the F-actin capping protein Cpal, which contributes to the positive selection of OT-1 cells, can be mediated by immunoproteasomes but not by constitutive proteasomes. Furthermore, repeated injection of $\beta 5$ i-deficient OT-1 mice with synthetic Cpa $1_{92-99}$ peptide rescued the positive selection of OT-1 cells, which emphasizes the role of $\beta 5 \mathrm{i}$ in this process.

Although this finding strongly supports the involvement of immunoproteasomes (and $\beta 5 \mathrm{i}$ in particular) in positive selection, it is difficult to reconcile the result with the failure to detect $\beta 5 \mathrm{i}$ mRNA and protein in cTECs from non-infected mice ${ }^{47}$ and with the recent finding that $\beta 5 \mathrm{i}$ is replaced by the CTEC-specific subunit $\beta 5$ t in these cells (see below).

\section{Thymoproteasomes in T cell selection} The interest in the role of immune-type proteasomes in TCR repertoire selection has recently been boosted by the discovery of a seventh active subunit of the 


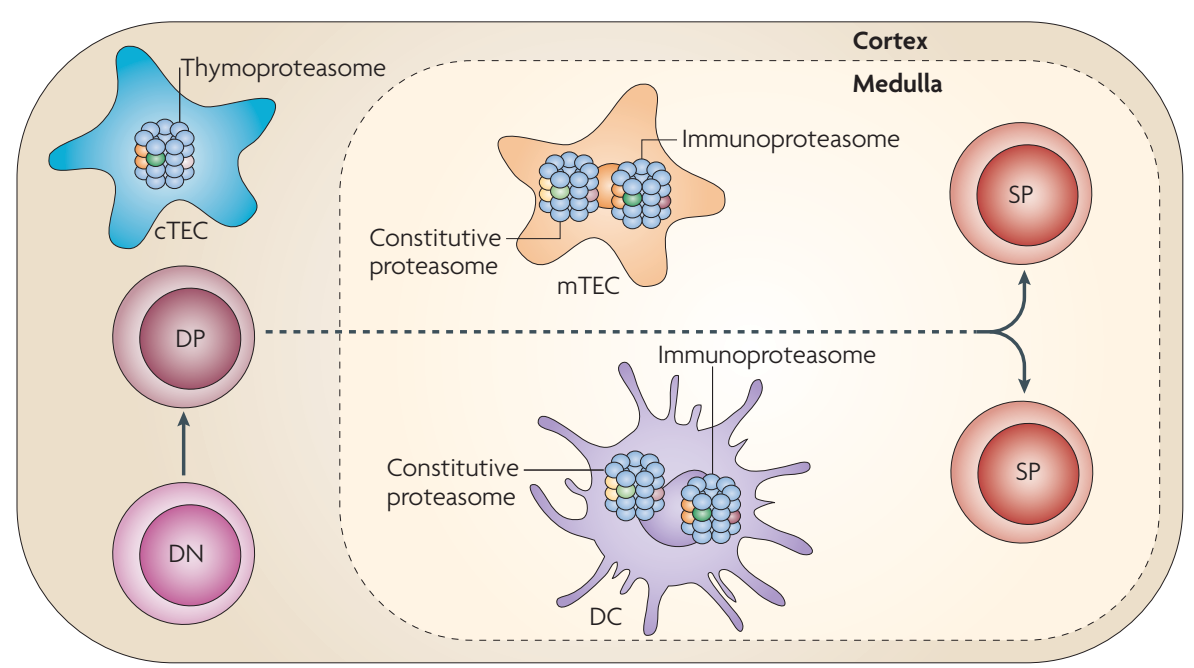

Figure $3 \mid$ Proteasomes in positive and negative selection in the thymus. Positive selection occurs at the double-positive (DP; $C D 4^{+} C D 8^{+}$) thymocyte stage and is mediated by cortical thymic epithelial cells (cTECs). These highly specialized antigen-presenting cells express a unique type of proteasome, known as the thymoproteasome, which contains the active site subunits $\beta 1 \mathrm{i}, \beta 2 \mathrm{i}$ and the cTEC-specific subunit $\beta 5$ t. Low-affinity interactions with the T cell receptor for the positive selection of CD8 singlepositive (SP) thymocytes probably rely on a spectrum of weak peptide-MHC class I ligands with hydrophilic carboxy-termini that are generated by the thymoproteasome. Medullary thymic epithelial cells (mTECs) and dendritic cells (DCs) mediate the negative selection of self-reactive thymocytes at the boundary of the cortex and medulla. Both cell types express high levels of immunoproteasomes containing the active site subunits $\beta 1 \mathrm{i}, \beta 2 \mathrm{i}$ and $\beta 5 \mathrm{i}$, as well as constitutive proteasomes containing $\beta 1$, $\beta 2$ and $\beta 5$. The negative selection of CD8 SP thymocytes relies on high-affinity peptide-MHC class I ligands and should involve peptides generated from self antigens by both types of proteasome that are encountered in the periphery (the constitutive proteasome and the immunoproteasome).

mammalian proteasome known as $\beta 5$ t, which is expressed exclusively by cTECs in mice $^{37}$ and humans ${ }^{49}$. $\beta 5$ t from mouse thymus lysates co-immunoprecipitates with $\beta 1 i$ and $\beta 2$ i, but not with $\beta 1$ or $\beta 2$ proteasome subunits $^{37}$. This $\beta 1 \mathrm{i}-\beta 2 \mathrm{i}-\beta 5 \mathrm{t}$-containing proteasome has been designated the thymoproteasome to distinguish it from the $\beta 1 \mathrm{i}-\beta 2 \mathrm{i}-\beta 5 \mathrm{i}$-containing immunoproteasome (FIG. 2). In contrast to the classical immunosubunits ( $\beta 1 \mathrm{i}$ and $\beta 2 \mathrm{i})$, expression of $\beta 5 \mathrm{t}$ is not induced by IFN $\gamma$. Ly5 $1^{+}$cTECs were shown to express low levels of the proteasome subunits $\beta 1, \beta 2, \beta 5$ and $\beta 5 i$, whereas $\beta 1 \mathrm{i}, \beta 2 \mathrm{i}$ and $\beta 5 \mathrm{t}$ were highly expressed ${ }^{37}$. This result is in conflict with an earlier report that showed that mouse cTECs express the constitutive proteasome subunits $\beta 1, \beta 2$ and $\beta 5$ and only express the immunosubunits $\beta 1$, $\beta 2 \mathrm{i}$ and $\beta 5 \mathrm{i}$ after infection or IFN $\gamma$ stimulation in $v i v o^{47}$. These disparate results could be explained by differences in infection status and cytokine levels in the mice or by the presence of other cell types in the cTEC preparations. Further investigation of the proteasome subunits that are expressed by cTECs from naive and infected mice is warranted to determine if inducing the expression of $\beta 5 \mathrm{i}$ can replace $\beta 5 \mathrm{t}$ in proteasome assembly in cTECs as it does for $\beta 5$ in other tissues.
Why does a highly specialized cell type that has evolved to mediate positive selection in the thymus require a unique $\beta 5$-type proteasome subunit? Examination of the S1 pocket of $\beta 5 \mathrm{t}$, which accommodates the amino acid directly before the polypeptide cleavage site, shows that it is lined with hydrophilic residues rather than the hydrophobic residues that are present in $\beta 5$ and $\beta 5 \mathrm{i}$ (REF. 37). This change in the S1 pocket decreases the chymotrypsinlike activity (cleavage after hydrophobic residues) of $\beta 5$ t by $60-70 \%$, and probably affects the pool of available ligands for MHC class I molecules that are generated. Peptides with hydrophilic C-termini (such as those produced by $\beta 5 \mathrm{t}$ ) are predicted to be poor ligands for MHC class I molecules, and although there are normal numbers of double-negative, double-positive and $\mathrm{CD} 4$ single-positive (SP) thymocytes in $\beta 5 \mathrm{t}$-deficient mice, the number of CD8 SP thymocytes and peripheral CD8 $8^{+} \mathrm{T}$ cells is decreased by $75 \%$ in these mice ${ }^{37}$ (FIG. 3).

The expression profile, biochemical properties and effect on thymic selection of $\beta 5 t$ are all consistent with a role for this proteasome subunit in the positive selection of $\mathrm{CD}^{+}$ $\mathrm{T}$ cells, but one finding has remained puzzling: low-affinity MHC class I ligands should negatively affect the cell surface expression of MHC class I molecules but this correlation was not found for $\beta 5 t$-deficient $\mathrm{CTECs}^{50}$. Why do $\beta 5$ t-deficient cTECs have such a marked deficit in positive selection when the level of MHC class I cell-surface expression is not changed? It was speculated that the $\beta 5 t$-dependent peptide repertoire of cTECs is better suited for positive selection and that the peptide repertoire on which positive selection occurs should be different from the peptide repertoire of negatively selecting $\mathrm{APCs}^{50}$. The requirement of a special peptide population for positive selection is not fully consistent with the demonstration that a single peptide can positively select a large and diverse TCR repertoire ${ }^{51}$. One possible explanation could be that cTECs have mechanisms to better stabilize MHC class I molecules complexed with low-affinity peptide ligands. Alternatively, the $\beta 5 t$-generated peptide ligands could achieve a higher affinity for MHC class I molecules by the use of MHC anchor positions other than the C-terminal position; an experimental determination of the dissociation rates of MHC class I ligands in $\beta 5$ t-expressing cells should clarify this issue. Together, these results show that it is probable that the production of low-affinity peptide ligands for MHC class I molecules underlies the phenotype of $\beta 5 t$-deficient mice, but there is some room for alternative mechanisms.

\section{Immunoproteasomes in T cell survival} In addition to its role in shaping the antigenic peptide repertoire presented by MHC class I molecules, the immunoproteasome might have other roles in regulating immune responses. As mentioned earlier, after adoptive transfer into wild-type mice, $\mathrm{T}$ cells from $\beta 1 \mathrm{i}$-deficient mice fail to proliferate in response to influenza virus infection, despite the robust proliferation of host $\mathrm{T}$ cells ${ }^{39}$. It has been argued that this could be attributed to the rejection of $\beta 1$ i-deficient donor $\mathrm{T}$ cells by the wild-type host because the donor cells present a different peptide repertoire on their MHC class I molecules ${ }^{52}$. An argument against such a rejection phenomenon is provided by the finding that skin from $\beta 5 i$-deficient mice transplanted onto wild-type mice was not rejected by the host ${ }^{53}$. Strikingly, when $\beta 2$ i-deficient $\mathrm{T}$ cells were transferred into LCMV-infected wild-type mice, they also did not survive $e^{44}$. As the cell surface expression level of MHC class I molecules is not altered in $\beta 1 \mathrm{i}$ - or $\beta 2 \mathrm{i}$-deficient mice and as no major change in the specificity of proteasomal cleavage could be observed in $\beta 2 i$-deficient mice ${ }^{44}$, we think that the disappearance of $\beta 1 \mathrm{i}$ - or 
$\beta 2 \mathrm{i}$-deficient $\mathrm{T}$ cells after transfer into virusinfected wild-type mice is not the result of rejection but instead reflects the requirement of these immunoproteasome subunits for the survival of $\mathrm{T}$ cells in a pro-inflammatory environment. Based on this notion, we postulate that the immunoproteasome could be a suitable drug target for the suppression of overactive $\mathrm{T}$ cell responses such as are found in many autoimmune diseases.

\section{$\beta 5 i$ inhibition blocks autoimmunity}

The data generated from knockout mice highlight two important aspects of the biology of the immunoproteasome: first, the inducible subunits of the immunoproteasome have non-redundant immunoregulatory functions; and second, compensatory mechanisms and altered proteasome structure in subunit-deficient cells might complicate the search for specific pathways that are regulated by the immunoproteasome. A need for specific inhibitors of immunoproteasome subunits is evident. Small molecule inhibitors of the proteasome have been used in research since 1994 (REF. 54) and the dipeptide boronate proteasome inhibitor bortezomib (Velcade; Millennium Pharmaceuticals) is used for the treatment of malignant diseases, such as multiple myeloma ${ }^{55}$. However, these inhibitors do not selectively target immunoproteasome subunits and, so far, their clinical use has been restricted to the treatment of cancer owing to drug side effects.

Recently, a cell-permeable ketoepoxidebased immunoproteasome inhibitor, designated PR-957, which selectively inhibits $\beta 5 \mathrm{i}$ in both human and mouse cells at concentrations that do not target other proteasome subunits, has been developed ${ }^{56}$. The selectivity of PR-957 was verified by its ability to downregulate MHC class I cell surface expression by $50 \%$ in wild-type but not $\beta 5$ i-deficient mice and to suppress the presentation of $\beta 5 \mathrm{i}$ dependent peptide epitopes, such as Uty ${ }_{246-254}$ (derived from the male minor antigen $\mathrm{HY}$ ) and LCMV GP33, without affecting the presentation of $\beta 5$ i-independent epitopes.

As PR-957 suppressed the presentation of the LCMV GP33 epitope in vivo, we investigated whether PR-957 could prevent diabetes in RIP-GP mice, which express a fragment of the LCMV glycoprotein epitope in $\beta$-islet cells under the control of the rat insulin promoter (RIP) and develop diabetes after infection with LCMV ${ }^{57}$. Treatment with PR-957 completely prevented the onset of disease after virus challenge in RIP-GP mice, highlighting the role of $\beta 5 \mathrm{i}$ in the production of the immunodominant LCMV GP33 epitope ${ }^{56}$, which could not be fully appreciated in experiments with $\beta 5 i-$ deficient mice most probably owing to the concomitant lack of $\beta 1 \mathrm{i}$ and $\beta 2 \mathrm{i}^{38}$.

The role of $\beta 5 \mathrm{i}$ in immune responses is not restricted to T cells. We found that selectively targeting $\beta 5 \mathrm{i}$ in human peripheral blood mononuclear cells (PBMCs) blocked the production of several pro-inflammatory cytokines, including interleukin-6 (IL-6), IL-23 and TNF ${ }^{56}$. The level of inhibition varied for each cytokine but was equivalent in PBMCs derived from healthy volunteers and from patients with active rheumatoid arthritis, which indicates that immunomodulation through targeting $\beta 5$ i might be feasible in patients with rheumatoid arthritis. The suppression of IL- 6 and IL-23 production is intriguing given that these cytokines have a crucial role in the development and/or maintenance of $T$ helper $17\left(\mathrm{~T}_{\mathrm{H}} 17\right)$ cells, which are involved in the pathogenesis of several autoimmune diseases, including rheumatoid arthritis, inflammatory bowel disease and psoriasis ${ }^{58}$. Interestingly, $\beta 2 \mathrm{i}$-deficient mice have been shown to be protected from dextran sulphate sodium-induced colitis, which is a T cell-independent mouse model of inflammatory bowel disease ${ }^{59}$. PR-957 suppressed the development of $\mathrm{T}_{\mathrm{H}} 1$ and $\mathrm{T}_{\mathrm{H}} 17$ cells in vitro from both mouse and human naive $T$ cells but did not affect the differentiation of regulatory $\mathrm{T}$ cells and $\mathrm{T}_{\mathrm{H}} 2$ cells $^{56}$ (E. Suzuki, C.J.K., M.B. and K. Kalim, unpublished observations).

We extended these in vitro data to show that PR-957 could block disease progression in mouse models of rheumatoid arthritis. Inhibition of $\beta 5 i$ suppressed disease symptoms in both collagen-induced arthritis (CIA) and collagen antibody-induced arthritis (CAIA) and was more effective than the soluble TNF antagonist etanercept ${ }^{56}$. PR-957 suppressed disease symptoms at doses of less than one-tenth the maximum tolerated dose, a therapeutic window that is not achievable with non-selective inhibitors. The efficacy of PR-957 in the T cell-independent CAIA model highlights the immunoregulatory role of $\beta 5$ i outside of antigen presentation that, consistent with in vitro data, involves multiple immune effector cell types. Therapeutic activity of PR-957 in mouse models of colitis, dermatitis and lupus has also been noted (T. Muchamuel, C.J.K., M.B. and M.G., unpublished observations).

\section{Conclusions and perspectives}

Despite the initial studies with immunoproteasome-deficient mice that indicated a mild phenotype, more recent studies involving pathogen infection models have shown that immunoproteasome subunits have distinct functions in pathogen elimination ${ }^{41,42}$. Further studies with both knockout mice and selective inhibitors will enable a more complete understanding of how the immunoproteasome and thymoproteasome shape the antigenic repertoire and regulate the CTL response to infectious agents. However, questions remain about the role of specific subunits in immune responses. It is unclear why $\beta 5 \mathrm{i}$ but not $\beta 1 \mathrm{i}$ or $\beta 2 \mathrm{i}$ deficiency decreases the cell surface expression of MHC class I molecules but the number of $\mathrm{CD} 8^{+} \mathrm{T}$ cells is decreased in $\beta 1 \mathrm{i}$ - or $\beta 2 \mathrm{i}$-deficient mice, but not $\beta 5 \mathrm{i}$-deficient mice. What are the pathways that are regulated by the immunoproteasome that underlie its role in the proliferative expansion of $\mathrm{T}$ cells in a proinflammatory environment? Are these pathways similar to the pathways that are regulated by $\beta 5$ i during cytokine production by $\mathrm{T}$ cells and monocytes?

The development of the specific $\beta 5 \mathrm{i}$ inhibitor PR-957 has opened new and interesting avenues of research into immunoproteasome biology. Further work is required to fully understand the unique role of the immunoproteasome in the induction and maintenance of inflammation. The suppression of $\mathrm{T}_{\mathrm{H}} 1$ and $\mathrm{T}_{\mathrm{H}} 17$ cell differentiation by PR-957 indicates that the immunoproteasome is a possible target for therapeutic intervention in several autoimmune diseases. However, it is unclear how the immunoproteasome is mechanistically involved in these processes. We propose that the immunoproteasome selectively processes a factor that is required for regulating cytokine production. Clearly, more research, including the development of selective inhibitors of $\beta 1 \mathrm{i}$ and $\beta 2 \mathrm{i}$, is required. Clinical investigation with PR-957 in autoimmune disease will bring the immunoproteasome forward as a drug target and extend these interesting preclinical findings. From subtle phenotypes in gene-deficient mice to a drug target in inflammatory diseases, the immunoproteasome is back in the limelight.

Marcus Groettrup and Michael Basler are at the Division of Immunology, Department of Biology, University of Constance, D-78, 457 Konstanz, Germany, and at the Biotechnology Institute Thurgau (BITg) at the University of Constance, $\mathrm{CH}-8,280$ Kreuzlingen, Switzerland.

Christopher J. Kirk is at Onyx Pharmaceuticals, Emeryville, California 94608, USA.

Correspondence to M.G. e-mail:Marcus.Groettrup@uni-konstanz.de 
1. Heath, W. R. et al. Cross-presentation, dendritic cell subsets, and the generation of immunity to cellular antigens. Immunol. Rev. 199, 9-26 (2004).

2. Hammer, G. E., Kanaseki, T. \& Shastri, N. The fina touches make perfect the peptide-MHC class I repertoire. Immunity 26, 397-406 (2007).

3. Löwe, J. et al. Crystal structure of the $20 \mathrm{~S}$ proteasome from the archaeon T. acidophilum at $3.4 \AA$ resolution. Science 268, 533-539 (1995)

4. Seemüller, E. et al. Proteasome from Thermoplasma acidophilum: a threonine protease. Science $\mathbf{2 6 8}$, 579-582 (1995).

5. Finley, D. Recognition and processing of ubiquitinprotein conjugates by the proteasome. Annu. Rev. Biochem. 78, 477-513 (2009).

6. Dubiel, W., Pratt, G., Ferrell, K. \& Rechsteiner, M. Purification of an $11 \mathrm{~S}$ regulator of the multicatalytic proteinase. J. Biol. Chem. 267, 22369-22377 (1992)

7. Chu-Ping, M., Slaughter, C. A. \& DeMartino, G. N. Identification, purification and characterization of a protein activator (PA28) of the 20S proteasome (Macropain). J. Biol. Chem. 267, 10515-10523 (1992).

8. Hendil, K. B., Khan, S. \& Tanaka, K. Simultaneous binding of PA28 and PA700 activators to $20 \mathrm{~S}$ proteasomes. Biochem. J. 332, 749-754 (1998)

9. Groettrup, M. et al. A role for the proteasome regulator PA28 $\alpha$ in antigen presentation. Nature 381, 166-168 (1996).

10. van Hall, T. et al. Differential influence on cytotoxic T lymphocyte epitope presentation by controlled expression of either proteasome immunosubunits or PA28. J. Exp. Med. 192, 483-494 (2000).

11. Murata, S. et al. Immunoproteasome assembly and antigen presentation in mice lacking both PA28 $\alpha$ and PA283. EMBO J. 20, 5898-5907 (2001).

12. Dick, T. P. et al. Coordinated dual cleavages by the proteasome regulator PA28 lead to dominant $\mathrm{MHC}$ ligands. Cell 86, 253-262 (1996).

13. Li, J. et al. Lysine 188 substitutions convert the pattern of proteasome activation by REG $\gamma$ to that of REGs $\alpha$ and $\beta$. EMBO J. 20, 3359-3369 (2001).

14. Whitby, F. G. et al. Structural basis for the activation of $20 \mathrm{~S}$ proteasomes by $11 \mathrm{~S}$ regulators. Nature 408 , 115-120 (2000).

15. Rammensee, H. G. Bachmann, J., Emmerich, N. P. N Bachor, O. A. \& Stevanovic, S. SYFPEITHI: database for MHC ligands and peptide motifs. Immunogenetics 50, 213-219 (1999).

16. Glynne, R. et al. A proteasome-related gene between the two $\mathrm{ABC}$ transporter loci in the class II region of the human MHC. Nature 353, 357-360 (1991).

17. Kelly, A. et al. Second proteasome-related gene in the human MHC class II region. Nature 353, 667-668 (1991).

18. Ortiz-Navarette, V. et al. Subunit of the $20 \mathrm{~S}$ proteasome (multicatalytic proteinase) encoded by the major histocompatibilty complex. Nature 353, 662-664 (1991)

19. Brown, M. G., Driscoll, J. \& Monaco, J. J. Structural and serological similarity of MHC-linked LMP and proteasome (multicatalytic proteinase) complexes. Nature 353, 355-357 (1991).

20. Aki, M. et al. Interferon- $\gamma$ induces different subunit organizations and functional diversity of proteasomes. J. Biochem. 115, 257-269 (1994)

21. Groettrup, M. et al. A third interferon- $\gamma$-induced subunit exchange in the $20 \mathrm{~S}$ proteasome. Eur. J. Immunol. 26, 863-869 (1996).

22. Nandi, D., Jiang, H. \& Monaco, J. J. Identification of MECL-1 (LMP-10) as the third IFN- $\gamma$-inducible proteasome subunit. J. Immunol. 156, 2361-2364 (1996).

23. Hisamatsu, H. et al. Newly identified pair of proteasomal subunits regulated reciprocally by interferon $\gamma$.J. Exp. Med. 183, 1-10 (1996).

24. Akiyama, K. et al. cDNA cloning and interferon $\gamma$ down-regulation of proteasomal subunits $X$ and $Y$. Science 265, 1231-1234 (1994).

25. Boes, B. et al. Interferon $\gamma$ stimulation modulates the proteolytic activity and cleavage site preference of
$20 \mathrm{~S}$ mouse proteasomes. J. Exp. Med. 179, 901-909 (1994).

26. Khan, S. et al. Immunoproteasomes largely replace constitutive proteasomes during an antiviral and antibacterial immune response in the liver. J. Immunol. 167, 6859-6868 (2001)

27. Barton, L. F., Cruz, M., Rangwala, R., Deepe, G. S. \& Monaco, J. J. Regulation of immunoproteasome subunit expression in vivo following pathogenic fungal infection. J. Immunol. 169, 3046-3052 (2002).

28. Groettrup, M. et al. Structural plasticity of the proteasome and its function in antigen processing. Crit. Rev. Immunol 21, 339-359 (2001).

29. Kloetzel, P. M. Antigen processing by the proteasome Nature Rev. Mol. Cell Biol. 2, 179-187 (2001).

30. Gaczynska, M., Rock, K. L. ¿ Goldberg, A. L. $\gamma$-Interferon and expression of MHC genes regulate peptide hydrolysis by proteasomes. Nature 365 264-267 (1993).

31 Driscoll, J., Brown, M. G. Finley, D. \& Monaco, J. J. MHC-linked LMP gene products specifically alter peptidase activities of the proteasome. Nature 365 peptidase activities
262-264 (1993).

32. Kuckelkorn, U. et al. Incorporation of major histocompatibility complex-encoded subunits LMP2 and LMP7 changes the quality of the 20S proteasome polypeptide processing products independent of interferon- $\gamma$. Eur. J. Immunol. 25, 2605-2611 (1995)

33. Groll, M. et al. Structure of $20 \mathrm{~S}$ proteasome from yeast at 2.4A resolution. Nature 386, 463-471 (1997).

34. Unno, M. et al. The structure of the mammalian $20 \mathrm{~S}$ proteasome at $2.75 \AA$ resolution. Structure 10 609-618 (2002).

35. Van Kaer, L. et al. Altered peptidase and viral-specific T cell response in LMP2 mutant mice. Immunity 1 , 533-541 (1994).

36. Fehling, $\mathrm{H}$. J. et al. MHC class I expression in mice lacking proteasome subunit LMP7. Science 265 1234-1237 (1994)

37. Murata, S. et al. Regulation of $\mathrm{CD} 8+\mathrm{T}$ cell development by thymus-specific proteasomes. Science $\mathbf{3 1 6}$, 1349-1353 (2007).

38. Basler, M., Youhnovski, N., van den Broek, M. Przybylski, M. \& Groettrup, M. Immunoproteasomes down-regulate presentation of a subdominant $\mathrm{T}$ cell epitope from lymphocytic choriomeningitis virus. epitope from lymphocytic choriomening

39. Chen, W. S., Norbury, C. C., Cho, Y. J., Yewdell, J. W. \& Bennink, J. R. Immunoproteasomes shape immunodominance hierarchies of antiviral $\mathrm{CD} 8^{+} \mathrm{T}$ cells at the levels of $\mathrm{T}$ cell repertoire and presentation of viral antigens. J. Exp. Med. 193, 1319-1326 (2001).

40. Nussbaum, A. K., Rodriguez-Carreno, M. P. Benning, N., Botten, J. \& Whitton, J. L. Immunoproteasome-deficient mice mount largely normal CD8+ T cell responses to lymphocytic choriomeningitis virus infection and DNA vaccination. J. Immunol. 175, 1153-1160 (2005).

41. Strehl, B. et al. Immunoproteasomes are essential for clearance of Listeria monocytogenes in nonlymphoid tissues but not for induction of bacteria-specific CD8 ${ }^{+}$ T cells. J. Immunol. 177, 6238-6244 (2006)

42. Tu, L. et al. Critical role for the immunoproteasome subunit LMP7 in the resistance of mice to Toxoplasma gondii infection. Eur. J. Immunol. 4 Nov 2009 (doi: 10.1002/eji.200939117).

43. Boehm, U., Klamp, T., Groot, M. \& Howard, J. C. Cellular responses to interferon- $\gamma$. Annu. Rev. Immunol. 15, 749-795 (1997).

44. Basler, M., Moebius, J., Elenich, L., Groettrup, M. \& Monaco, J. J. An altered T cell repertoire in MECL-1. deficient mice. J. Immunol. 176, 6665-6672 (2006)

45. Caudill, C. M. et al. T cells lacking immunoproteasome subunits MECL-1 and LMP7 hyperproliferate in response to polyclonal mitogens. J. Immunol. 176 4075-4082 (2006).

46. Zaiss, D. M. W., de Graaf, N. \& Sijts, A. J. A. M The proteasome immunosubunit multicatalytic endopeptidase complex-like 1 is a T-cell-intrinsic factor influencing homeostatic expansion. Infec. Immun. 76, 1207-1213 (2008).
47. Nil, A., Firat, E., Sobek, V., Eichmann, K. ¿ Niedermann, G. Expression of housekeeping and immunoproteasome subunit genes is differentially regulated in positively and negatively selecting thymic stroma subsets. Eur. J. Immunol. 34, 2681-2689 (2004).

48. Osterloh, P. et al. Proteasomes shape the repertoire of $T$ cells participating in antigen-specific immune responses. Proc. Natl Acad. Sci. USA 103 5042-5047 (2006)

49. Tomaru, U. et al. Exclusive expression of proteasome subunit $\beta 5$ t in the human thymic cortex. Blood 113 5186-5191 (2009)

50. Murata, S. Takaharna, Y. \& Tanaka, K. Thymoproteasome: probable role in generating positively selecting peptides. Curr. Opin. Immunol. 20, 192-196 (2008)

51. Ignatowicz, L., Kappler, J. \& Marrack, P. The repertoire of T cells shaped by a single MHC/peptide ligand. Cell 84, 521-529 (1996).

52. Pang, K. C. et al. Immunoproteasome subunit deficiencies impact differentially on two immunodominant influenza-virus-specific $\mathrm{CD} 8+\mathrm{T}$ cell responses. J. Immunol. 177, 7680-7688 (2006).

53. Toes, R. E. M. et al. Discrete cleavage motifs of constitutive and immunoproteasomes revealed by quantitative analysis of cleavage products. J. Exp. Med. 194, 1-12 (2001).

54. Rock, K. L. et al. Inhibitors of the proteasome block the degradation of most cell proteins and the generation of peptides presented on MHC class I molecules. Cell 78, 761-771 (1994)

55. Bennett, M. K. \& Kirk, C. J. Development of proteasome inhibitors in oncology and autoimmune diseases. Curr. Opin. Drug Discov. Devel. 11, 616-625 (2008).

56. Muchamuel, T et al. A selective inhibitor of the immunoproteasome subunit LMP7 blocks cytokine production and attenuates progression of experimental arthritis. Nature Med. 15, 781-787 (2009).

57. Ohashi, P. S. et al. Ablation of 'tolerance' and induction of diabetes by virus infection in viral antigen transgenic mice. Cell 65, 305-317 (1991).

58. Ouyang, W. Kolls, J. K. \& Zheng, Y. The biological functions of Thelper 17 cell effector cytokines in inflammation. Immunity 28, 454-467 (2008).

59. Fitzpatrick, L. R., Khare, V., Small, J. S. ¿ Koltun, W. A Dextran sulfate sodium-induced colitis is associated with enhanced low molecular mass polypeptide 2 (LMP2) expression and is attenuated in LMP2 knockout mice. Digest Dis. Sci. 51, 1269-1276 (2006).

Acknowledgements

We thank J. Moebius, K. W. Kalim, E. Suzuki and T. Muchamuel for the communication of data before they were accepted for publication. This work was supported by grants from the German Research Foundation (DFG) - GR 1517/4-2 and
GR1517/5-1 - and the Graduate School Chemical Biology at the University of Constance, Germany.

\section{Competing interests statement}

The authors declare no competing financial interests. 